Le présent article témoigne de l'engagement des généralistes, des instituts de médecine de famille et des associations professionnelles dans le domaine des soins palliatifs. En cette période de pandémie de COVID-19, cet engagement revêt une importance toute particulière. II reste juste à espérer que les conditions-cadres continueront de s'améliorer afin que la médecine palliative puisse être mise en œuvre pour ce qu'elle est. Il semblerait que ce n'est pas l'engagement qui manque de la part des médecins et des professionnels de la santé.

Dr méd. Carlos Beat Quinto, membre du Comité central de la FMH, responsable du département Santé publique et professions de la santé

\title{
Soins palliatifs et médecine de famille
}

\author{
Christoph Cina ${ }^{a}$, Andreas Lischer ${ }^{a}$, Christoph Merloa, Christian Studera, Linda Hadorn ${ }^{b}$ \\ ${ }^{a}$ Groupe professionnels médecins palliative ch, Institut für Hausarztmedizin \& Community Care Lucerne (IHAM \& CC); ${ }^{b}$ Division Santé publique de la FMH
}

\section{De l'engagement à la mise en œuvre concrète}

Les grandes sociétés de médecins de premier recours comme la SSMIG et mfe reconnaissent conjointement avec palliative ch que les patients nécessitant des soins palliatifs devraient être pris en charge au niveau régional dans un cadre interprofessionnel. Ils veillent à ce que leurs membres connaissent les recommandations et les outils pratiques des soins palliatifs, tels que les tables rondes, les plans de soins et les évaluations selon le modèle SENS [1], et à ce que la charge de travail nécessaire à la prise en charge des patients en soins palliatifs soit correctement prise en compte par le tarif [2].

Une collaboration interprofessionnelle réussie se fonde sur la dignité (inviolable) de l'individu. Le constat que chacun, dans sa vulnérabilité, peut avoir besoin d'une aide extérieure en fin de vie et qu'il a droit à une vie digne jusqu'à la mort, devrait inciter à une nouvelle culture du soin (communauté de soins). Cette culture implique une collaboration interprofessionnelle pour le bien du patient, au sens d'une médecine centrée sur les patients.

On pourrait aussi parler d'une culture de soin communautaire à la croisée des différents «univers» impliqués de manière responsable et en toute confiance dans la prise en charge.

\section{Médecine de famille et communauté de soins}

Le médecin de famille suit ses patients pendant des années; il est donc aussi généralement leur premier interlocuteur pour les questions de santé. Il effectue également des visites à domicile, connaît les infrastructures locales et dispose d'un vaste réseau de relations. Cette conception de la profession confère au médecin de famille une valeur ajoutée irremplaçable susceptible d'être apportée à la collaboration interprofessionnelle.

\section{Conditions requises pour une collabora- tion interprofessionnelle réussie}

Une collaboration fructueuse repose sur des valeurs communes telles que la volonté de découvrir les compétences et aptitudes des différentes professions et de les aborder comme un enrichissement et non dans un esprit de concurrence. Les relations de travail se caractérisent par une estime mutuelle, une collaboration d'égal à égal avec des processus et des actions synchronisés. L'objectif central est de se concentrer sur les besoins, les symptômes et les problèmes des patients concernés et de leurs proches. Pour la prise en charge concrète des patients, il convient de clarifier les compétences, les aptitudes et les responsabilités lors de "tables rondes». Une collaboration réussie se fonde sur le consensus, aussi bien pour les normes, recommandations et standards que pour les outils de travail.

\section{Consensus pour les outils de travail et les standards}

Le modèle SENS s'est progressivement généralisé comme un instrument d'évaluation en médecine de premier recours [1]. Les compétences «Prise de décision» et "Gestion des symptômes» visent à renforcer l'autonomie dans le but d'encourager l'autodétermination et la capacité à décider. Les compétences «Réseau» et «Soutien des proches» favorisent l'intégrité sociale et procurent un sentiment de sécurité avec la sensation «d'être entre de bonnes mains».

Ces compétences peuvent être représentées de manière modulaire dans un plan de soins [3] qui servira 
de feuille de route à l'équipe interprofessionnelle. L'objectif est le respect de l'humain et de sa dignité.

Les patients polymorbides sont souvent soumis à une polypharmacie étendue. Un plan de médication uniforme qui supprime tous les médicaments non indiqués améliore d'une part la sécurité médicamenteuse et réduit d'autre part la lourdeur du traitement. Une plate-forme [3] interprofessionnelle numérique à laquelle participent tous les groupes professionnels impliqués permet d'améliorer considérablement la communication.

\section{Nombre important de visites à domicile}

Selon une enquête nationale menée par le réseau Sentinella (données IHAM \& CC pas encore publiées), $84 \%$ des médecins de famille interrogés proposent des visites à domicile représentant 1,8\% de toutes les consul-

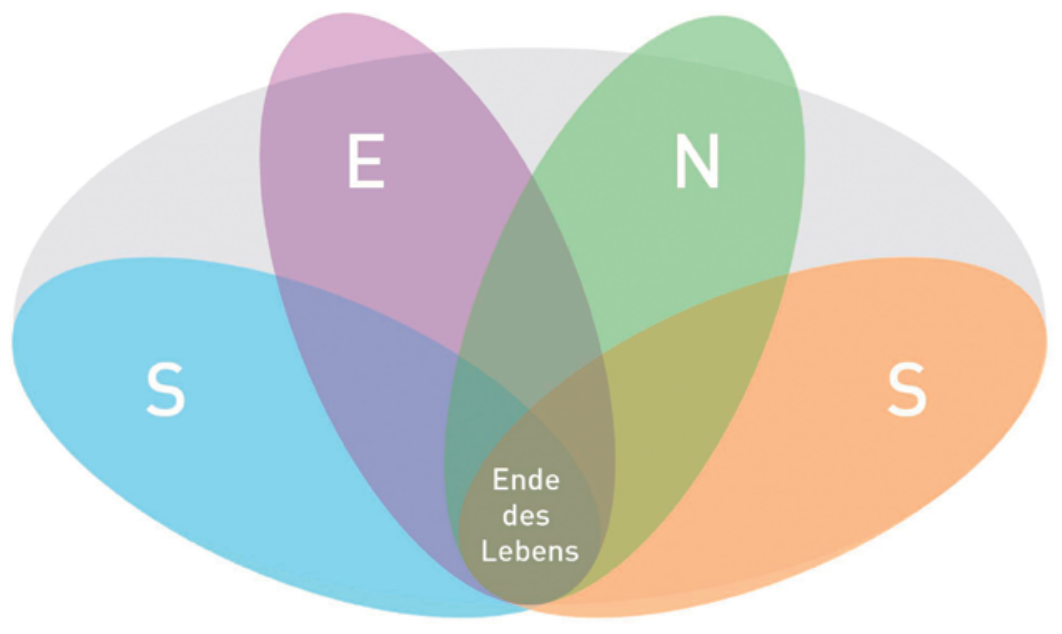

Grundlagen

Transversale Kompetenzen, die sowohl für das Symptom-Management, die Entscheidungsfindung. das Netzwerk und den Support als Grundvoraussetzungen gelten damit Palliative Care/die Palliativversorgung erfolgreich umgesetzt werden kann.

Symptom-Management

Beste Möglichkeiten der Symptombehandlung und Empowerment zur Selbsthilfe in der Symptombehandlung.

\section{Entscheidungsfindung}

Definition der eigenen Ziele und Priorităten, schrittweise, selbstgesteuerte Entscheidungsfindung und präventive Planung für mögliche Komplikationen.

\section{Ende des Lebens}

Hat seine eigenen Herausforderungen, die sowohl das Symptom-Management, die Entscheidungsfindung, das Netzwerk und den Support betreffen. Die Entscheidungsfindung wird jedoch meist als am schwerwiegendsten empfunden.

Netzwerk

Aufbau eines Betreuungsnetzes unter Zusammenführung von ambulanten (Betreuung zuhause) und stationären Strukturen,

\section{Support}

Aufbau von Unterstützungssystemen für die Angehörigen, auch über den Tod hinaus: Unterstützungmöglichkeit auch für die beteiligten Fachpersonen.

\section{Un nouveau CAS Palliative Care}

\section{à Lucerne}

Depuis I'automne 2019, I'Université de Lucerne propose une nouvelle formation continue, un CAS en soins palliatifs, en coopération avec I'Hôpital cantonal de Lucerne. L'une des journées du premier module "Médecine palliative et accompagnement" est consacrée aux "soins palliatifs en médecine de famille et soins palliatifs à domicile». Après un exposé interactif de plusieurs heures et une introduction diversifiée au thème (Christoph Cina), I'Institut de médecine familiale et Community Care Lucerne organise l'après-midi trois ateliers parallèles.

tations. 50\% de ces visites ont lieu dans des institutions comme des EMS. La plupart des médecins interrogés juge les visites à domicile importantes ou très importantes. Ils les motivent principalement par leur désir de fournir un service au patient.

\section{Ateliers dans le cadre du CAS Palliative Care à Lucerne}

Les ateliers suivants mettent en lumière différents points forts des soins palliatifs en médecine de famille sur la base d'exemples concrets.

\section{"Gestion des symptômes dans l'activité du médecin de famille" (Andreas Lischer)} Le premier «S» du modèle SENS désigne les symptômes et reflète souvent aussi la "première» inquiétude de nos patients dans leur dernière phase de vie: «Vais-je suffoquer, ressentir de fortes douleurs, souffrir de confusion mentale?» Un plan de médication d'urgence réfléchi et compréhensible, qui reflète une connaissance fondée de la situation actuelle et de la planification future, permet de désamorcer ces questions et d'instaurer la confiance. Mais il est indispensable que ce plan fonctionne également la nuit et le week-end si l'on veut éviter des hospitalisations non souhaitées et inutiles.

En cas d'évolution rapide de la situation, la présence mentale et physique du médecin de famille et sa disponibilité par téléphone sont souvent requises. Par ailleurs, quelques instruments peuvent lui être utiles l'oxymètre de pouls ou un laboratoire de praticien facilitent la prise de décision ou la détermination $\mathrm{du}$ dosage dans certaines situations. Des outils électroniques l'assistent par exemple pour la rotation d'opiacés ou la sécabilité et la possibilité de broyer les comprimés. Mais dans la plupart des cas, le bon sens, l'intuition clinique et le professionnalisme suffisent à prendre une décision raisonnable. 


\section{«Dignité et démence - un défi particulier» (Christian Studer)}

La proportion de personnes de plus de 85 ans souffrant de démence représente déjà un pourcentage à deux chiffres. Il n'est pas rare que les soins palliatifs durent des mois, voire des années. La capacité de discernement diminue progressivement. En présence d'une évolution démentielle, la jurisprudence suisse a décidé de renverser la charge de la preuve: celle-ci incombe à celui qui affirme que le patient possède une capacité de discernement [4]. Il faut donc prouver la capacité partielle de discernement pour certaines questions spécifiques.

La prise en charge n'est pas axée sur les déficits, mais sur les ressources afin d'encourager l'autonomie résiduelle, la sécurité, l'estime de soi et la dignité des patients atteints de démence. Il est essentiel de préserver cette autonomie le plus longtemps possible tout en évaluant et respectant les possibilités d'activité du patient. L'effondrement des passerelles vers le passé peuvent faire surgir un nouveau système de valeurs susceptible de brusquer l'entourage. Les personnes chargées de l'accompagnement doivent donc faire preuve de bienveillance en s'efforçant d'équilibrer le maintien d'une autonomie résiduelle et la prise en charge des soins.

"Chances et risques de la médecine palliative dans le même bateau que les proches"

(Christoph Merlo)

Il est intéressant de noter que tant les proches que les professionnels de santé (corps médical et infirmier) se considèrent comme des acteurs clés dans la coordina- tion des soins palliatifs à domicile [5]. Il est donc indispensable de clarifier les rôles et de se soutenir mutuellement afin de parvenir à une prise en charge adéquate dans les situations de douleur difficile à contrôler, d'incontinence fécale et urinaire ou de tumeurs excrétrices. Une attention particulière doit être accordée aux ressources personnelles et sociales des proches en fonction desquelles le personnel soignant, le médecin de famille et les autres membres de l'équipe de soins apporteront leur soutien.

\section{Comment réussir la mise en œuvre concrète?}

Les soins palliatifs devraient faire partie intégrante de la formation des médecins de premier recours et de l'offre de formation continue universitaire. L'apprentissage à partir d'exemples positifs (modèles de bonne pratique) convient particulièrement aux cercles de qualité interdisciplinaires, interprofessionnels ou de médecine de famille. Dans l'idéal, c'est ainsi que pourront être formées les communautés de soins sur le terrain.

\section{Références}

1 Eychmüller Steffen. SENS macht Sinn-Der Weg zu einer neuen Assessment-Struktur in der Palliative Care. Ther Umsch. 2012 fév.;69(2):87-90.

2 Sind wir Hausärzte bereit für Palliative Care? PHC. 2020;20(2):77-8. 3 https://www.palliative-so.ch/Angebote/Arbeitsinstrumente-fuerFachpersonen/PKuEl/

4 https://www.unilu.ch/fileadmin/fakultaeten/rf/aebi/dok/Jusletter Urteilsunfaehiger Patient.pdf

5 Haute école spécialisée du Nord-Ouest de la Suisse, Haute école de psychologie appliquée. Rapport de projet «Palliative Care in der Schweiz - die Sicht der Leistungserbringenden» (PNR 74).

\section{Connectez-vous à myFMH}

Changez votre adresse sous «Données personnelles»

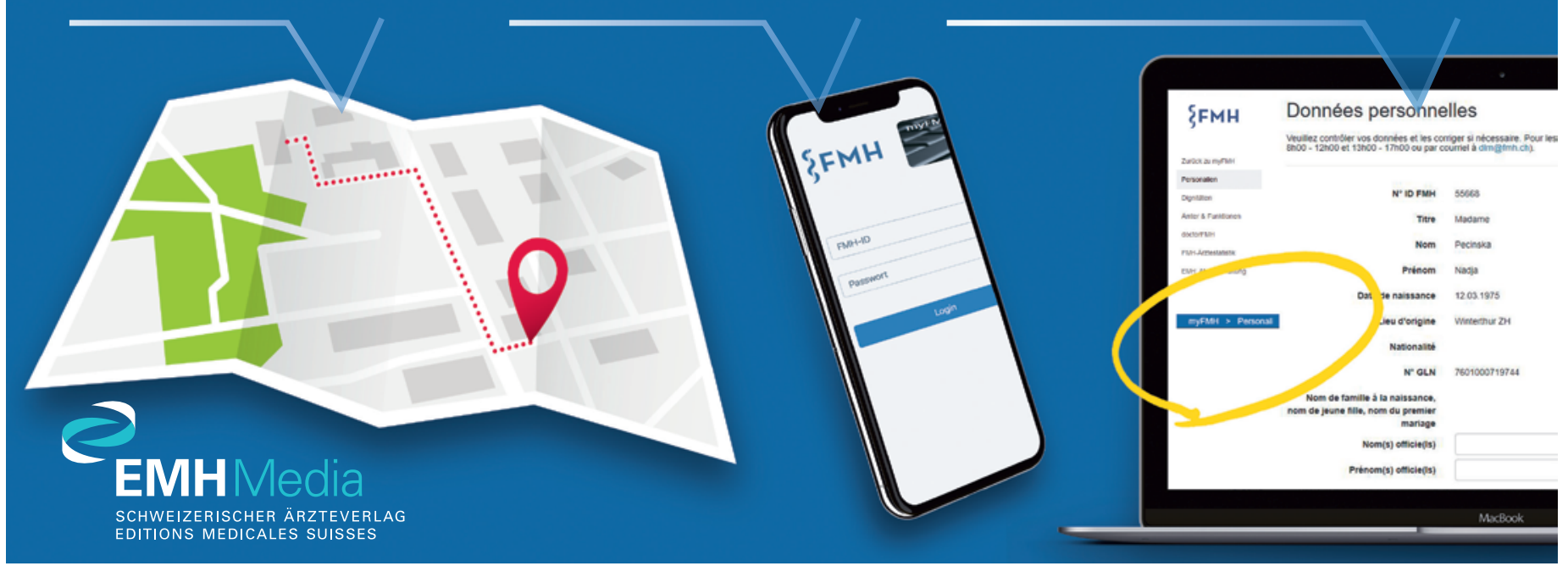

